\title{
Kinetic modulational instability of broadband dispersive Alfvén waves in plasmas
}

\author{
P. K. S H U K L A ${ }^{1,2,3,4,5}$, N I T I N S H U K L A ${ }^{1,6}$ \\ and L. ST E N F L O ${ }^{1,7}$ \\ ${ }^{1}$ Institut für Theoretische Physik IV and Centre for Plasma Science and Astrophysics, \\ Fakultät für Physik und Astronomie, Ruhr-Universität Bochum, \\ D-44780 Bochum, Germany \\ (ps@tp4.rub.de,ns@tp4.rub.de) \\ ${ }^{2}$ Max-Planck Institut für extraterrestrische Physik, D-45741 Garching, Germany \\ ${ }^{3}$ CCLRC Centre for Fundamental Physics, Rutherford Appleton Laboratory, Chilton, \\ Didcot, Oxon OX11 OQX, UK \\ ${ }^{4}$ SUPA Department of Physics, University of Strathclyde, Glasgow G4 ONG, UK \\ ${ }^{5}$ GoLP/Centro de Física de Plasmas, Instituto Superior Técnico, \\ 1096 Lisboa Codex, Portugal \\ ${ }^{6}$ Department of Physics, K. N. Government Postgraduate College, \\ Gyanpur, Bhadohi 221304, U.P., India \\ ${ }^{7}$ Department of Physics, Umeå University, SE-90187 Umeå, Sweden \\ (lennart.stenflo@physics.umu.se)
}

(Received 20 October 2006)

\begin{abstract}
We consider a kinetic modulational instability of broadband (random phase) magnetic-field-aligned circularly polarized dispersive Alfvén waves in plasmas. By treating random phase Alfvén waves as quasi-particles, we consider their nonlinear interactions with ion quasi-modes within the framework of the wavekinetic and Vlasov descriptions. A nonlinear dispersion relation governing such interactions is derived and analyzed. An explicit expression for the kinetic modulational instability growth rate is presented. Our results can be of relevance to the nonlinear propagation of incoherent Alfvén waves, which have been frequently observed in interstellar media, in the solar corona and in the solar wind, as well as in the foreshock regions of planetary bow-shocks and laboratory plasmas.
\end{abstract}

Dispersive Alfvén waves (DAWs) are of fundamental importance in astrophysical, space and laboratory plasmas [1-8]. They can be either circularly polarized electromagnetic waves, or an admixture of electrostatic and electromagnetic fields. The dispersion of low-frequency (in comparison with the ion gyrofrequency) circularly polarized DAW s comes from the ion inertia [3], while that of kinetic (inertial) Alfvén waves $[9,10]$ arises from the ion thermal gyroradius/ion polarization (the parallel electron inertia) effect. Large-amplitude DAWs, which can be excited by electron and proton beams, are capable of energizing charged particles and producing electron and ion heating in magnetoplasmas $[11,12]$. 
Large-amplitude coherent non-dispersive and DAWs are subjected to a great variety of nonlinear effects $[2,3,9,13-22]$. The latter include the three-wave decay $[13-16,22]$ and modulational instabilities [18-21]. However, the coherent wavewave and wave-particle $[15,21]$ interactions are inappropriate when the DAWs have random phases/broadband spectra.

In this letter, we consider the kinetic modulational instability of broadband righthand circularly polarized DAWs propagating along the external magnetic field $\hat{\mathbf{z}} B_{0}$ in a plasma, where $\hat{\mathbf{z}}$ is the unit vector along the $z$-axis in a Cartesian coordinate system and $B_{0}$ is the strength of the magnetic field. The wave magnetic field is given by $B_{k}=\left(B_{x}+i B_{y}\right) \exp \left(-i \omega_{k} t+i k z\right)$, where $B_{x}\left(B_{y}\right)$ is the $x(y)$ component of the wave magnetic field, and the wave frequency and wavenumber are related by

$$
\omega_{k} \approx k V_{\mathrm{A}}\left(1+k V_{\mathrm{A}} / 2 \omega_{c \mathrm{i}}\right),
$$

where $V_{\mathrm{A}}=B_{0} / \sqrt{4 \pi n_{\mathrm{i}} m_{\mathrm{i}}}$ and $\omega_{c \mathrm{i}}=e B_{0} / m_{\mathrm{i}} c$ are the Alfvén speed and the ion gyrofrequency, respectively, $n_{\mathrm{i}}$ is the ion number density, $m_{\mathrm{i}}$ is the ion mass, $e$ is the magnitude of the electron charge and $c$ is the speed of light in vacuum. We note that the wave dispersion in (1) comes from the finite frequency correction, and that $\omega \ll \omega_{\text {ci }}$.

We treat the DAWs, given by (1), as quasi-particles. The latter obey the Liouville equation $[23,24]$

$$
\frac{\partial I_{k}}{\partial t}+V_{\mathrm{g}} \frac{\partial I_{k}}{\partial z}+F_{k} \frac{\partial I_{k}}{\partial k}=0
$$

where $I_{k}=\left|B_{k}\right|^{2} / 8 \pi$ is the Alfvén wave energy density, $V_{\mathrm{g}}=\partial \omega_{k} / \partial k=V_{\mathrm{A} 0}+$ $k V_{\mathrm{A} 0}^{2} / \omega_{c \mathrm{i}}$ is the group velocity of the Alfvén wavepacket, and $V_{\mathrm{A} 0}=B_{0} / \sqrt{4 \pi n_{0} m_{\mathrm{i}}}$. The force exerted due to the background ion number density perturbation $n_{1}$ $\left(n_{1} \ll n_{0}\right.$, where $n_{0}$ is the unperturbed ion number density) on the Alfvén quasiparticles is

$$
F_{k}=-\frac{\partial \omega_{k}}{\partial z}=\frac{k V_{\mathrm{A} 0}}{2 n_{0}} \frac{\partial n_{1}}{\partial z} .
$$

The perturbed ion number density is

$$
n_{1}=\int d u f_{1}(t, z),
$$

which requires the knowledge of the perturbed ion distribution function $f_{1}(t, z)$ in the presence of the Alfvén quasi-particles. Using a hybrid approach, we start with the collisionless ion Vlasov equation

$$
\frac{\partial f_{1}}{\partial t}+u \frac{\partial f_{1}}{\partial z}+\frac{e}{m_{\mathrm{i}}}\left(E_{z}+\frac{1}{c}\left\langle\mathbf{v}_{k} \times \mathbf{B}_{k}\right\rangle \cdot \hat{\mathbf{z}}\right) \frac{\partial f_{0}}{\partial u}=0,
$$

where $E_{z}$ is the magnetic-field-aligned electric field associated with the ion quasimode, the angular bracket denotes the ensemble average over the Alfvén quasiparticles, $u$ is the $z$ component of the ion velocity, $\mathbf{v}_{k}$ is the ion velocity in the dispersive Alfvén wave fields and $f_{0}$ is the unperturbed ion velocity distribution function. The inertialess electron equation of motion

$$
E_{z}+\frac{1}{c}\left\langle\mathbf{v}_{k} \times \mathbf{B}_{k}\right\rangle \cdot \hat{\mathbf{z}}=-\frac{1}{n_{0} e} \frac{\partial}{\partial z}\left(\sum_{k} I_{k}+T_{\mathrm{e}} n_{1}\right)
$$

determines the parallel electric field $E_{z}$. Here $T_{\mathrm{e}}$ is the electron temperature. The quasi-neutrality condition for the ion quasi-mode has been invoked in (6). Hence, 
from (5) and (6) we have

$$
\frac{\partial f_{1}}{\partial t}+u \frac{\partial f_{1}}{\partial z}-\frac{1}{\rho} \frac{\partial}{\partial z}\left(\sum_{k} I_{k}+T_{\mathrm{e}} n_{1}\right) \frac{\partial f_{0}}{\partial u}=0
$$

where $\rho=n_{0} m_{\mathrm{i}}$ is the ion mass density.

Supposing that $I_{k}=I_{k 0}+I_{k 1} \exp (-i \Omega t+i K z)$, where $I_{k 1} \ll I_{k 0}$ and where $\Omega(K)$ is the frequency (wavenumber) of the ion quasi-mode, we obtain from (2)

$$
I_{k 1}=\frac{K V_{\mathrm{A} 0}}{2} \frac{k \partial I_{k 0} / \partial k}{\left(\Omega-K V_{\mathrm{A}}-k V_{G}\right)} \frac{n_{1}}{n_{0}},
$$

where $V_{G}=K V_{\mathrm{A} 0}^{2} / \omega_{c \mathrm{i}}$.

Furthermore, supposing that $f_{1}(t, u)$ is proportional to $\exp (-i \Omega t+i K z)$, we obtain from (7)

$$
f_{1}=-\frac{K}{\rho} \frac{\partial f_{0} / \partial u}{(\Omega-K u)}\left(\sum_{k} I_{k 1}+T_{\mathrm{e}} n_{1}\right) .
$$

Multiplying both sides of (9) by $d u$ and integrating over the velocity space, we obtain by using (4)

$$
n_{1}\left(1+\frac{K C_{\mathrm{s}}^{2}}{n_{0}} \int \frac{d u \partial f_{0} / \partial u}{\omega-K u}\right)+\frac{K}{\rho} \int \frac{d u \partial f_{0} / \partial u}{\Omega-K u} \sum_{k} I_{k 1}=0,
$$

where $C_{\mathrm{s}}=\left(T_{\mathrm{e}} / m_{\mathrm{i}}\right)^{1 / 2}$ is the ion sound speed.

Inserting $I_{k 1}$ from (8) into (10) we thus obtain the dispersion relation

$$
1+\frac{K C_{\mathrm{s}}^{2}}{n_{0}} \int \frac{d u \partial f_{0} / \partial u}{\Omega-K u}+\frac{K^{2} V_{\mathrm{A} 0}}{2 \rho n_{0}} \int \frac{d u \partial f_{0} / \partial u}{\Omega-K u} \sum_{k} \frac{k \partial I_{k 0} / \partial k}{\Omega_{0}-k V_{G}}=0,
$$

where $\Omega_{0}=\Omega-K V_{\mathrm{A} 0}$. Assuming a Maxwellian ion distribution function

$$
f_{0}=\frac{n_{0}}{\sqrt{2 \pi} V_{\mathrm{T}}} \exp \left(-u^{2} / 2 V_{\mathrm{T}}^{2}\right)
$$

where $V_{\mathrm{T}}=\left(T_{\mathrm{i}} / m_{\mathrm{i}}\right)^{1 / 2}$ is the ion thermal speed and $T_{\mathrm{i}}$ is the ion temperature, we obtain from (11)

$$
1+\frac{T_{\mathrm{e}}}{T_{\mathrm{i}}} W\left(\xi_{\mathrm{i}}\right)+\frac{K V_{\mathrm{A} 0} W\left(\xi_{\mathrm{i}}\right)}{2 V_{G} n_{0} T_{\mathrm{i}}} \frac{L}{2 \pi} \int \frac{d k k \partial I_{k 0} / \partial k}{\left(k-\Omega_{0} / V_{G}\right)}=0,
$$

where the length of the system is $L$, and the $W$ function is [25]

$$
W\left(\xi_{\mathrm{i}}\right)=\frac{1}{\sqrt{2 \pi}} \int \frac{d \xi \xi \exp \left(-\xi^{2} / 2\right)}{\xi-\xi_{\mathrm{i}}}
$$

with $\xi_{\mathrm{i}}=\Omega / K V_{\mathrm{T}}$.

We now choose

$$
I_{k 0}=\frac{I_{00}}{\sqrt{2 \pi} k_{\mathrm{w}} L} \exp \left[-\left(k-k_{0}\right)^{2} / 2 k_{\mathrm{w}}^{2}\right]
$$

where $I_{00}$ is the maximum Alfvén wave magnetic field energy density corresponding to a mean wavenumber $k_{0}$, and $k_{\mathrm{w}}$ represents the width of the DAW spectrum. Substituting for $I_{k 0}$ from (14) into (13) we finally obtain the desired dispersion relation

$$
1+\frac{T_{\mathrm{e}}}{T_{\mathrm{i}}} W\left(\xi_{\mathrm{i}}\right)-\frac{\left(\Omega-K V_{\mathrm{A} 0}\right) K V_{\mathrm{A} 0} W\left(\xi_{\mathrm{i}}\right)}{k_{\mathrm{w}}^{2} V_{G}^{2}} \frac{I_{00}}{8 \pi n_{0} T_{\mathrm{i}}} W\left(\xi_{0}\right)=0
$$


where $\xi_{0}=\left[\Omega-\left(K V_{\mathrm{A} 0}+k_{0} V_{G}\right)\right] / k_{\mathrm{w}} V_{G}$. For $\xi_{0} \gg 1$, (15) reduces to

$$
\left[\Omega-\left(K V_{\mathrm{A} 0}+k_{0} V_{G}\right)\right]^{2}=-\left(\Omega-K V_{\mathrm{A} 0}\right) K V_{\mathrm{A} 0} \frac{W\left(\xi_{\mathrm{i}}\right)}{\left[1+\sigma W\left(\xi_{\mathrm{i}}\right)\right]} \frac{I_{00}}{8 \pi n_{0} T_{\mathrm{i}}},
$$

where $\sigma=T_{\mathrm{e}} / T_{\mathrm{i}}$. Letting $\Omega=K V_{\mathrm{A} 0}+k_{0} V_{G}+i \gamma \equiv \Omega_{r}+i \gamma$, where $\gamma \ll \Omega_{r}$, we obtain from (16) the growth rate

$$
\gamma=\left(k_{0} V_{G} K V_{\mathrm{A} 0}\right)^{1 / 2}(\operatorname{Re} Q)^{1 / 2}\left(\frac{I_{00}}{8 \pi n_{0} T_{\mathrm{i}}}\right)^{1 / 2},
$$

where $Q=W\left(\xi_{r}\right) /\left[1+\sigma W\left(\xi_{r}\right)\right]$ with $\xi_{r}=\Omega_{r} / K V_{\mathrm{T}}$. Equation (15), together with (17), is the main result of this paper.

In summary, we have considered the nonlinear propagation of a broadband magnetic-field-aligned right-hand circularly polarized DAW in an electron-ion magnetoplasma. The dynamics of the broadband DAW has been treated by a wave kinetic equation, considering DAW propagation in a slowly varying medium containing ion density ripples (ion quasi-modes). The latter, which are obtained from the ion Vlasov equation and the parallel component of the ion momentum equation, are coupled with the random phase DAW via the ponderomotive force effect. The governing equations have been linearized around the equilibrium state to obtain a new dispersion relation, which admits a kinetic modulational instability of broadband DAWs in magnetized plasmas. In conclusion, we stress that the present result is important for understanding the stimulated scattering of incoherent (random phase) DAWs of ion quasi-modes in astrophysical [7] and laboratory plasmas [22]. Specifically, the present mechanism suggests a novel nonlinear scenario for the dissipation of DAW energy in magnetized plasmas.

\section{Acknowledgement}

This research was initiated while the authors were attending the International Workshop on Frontiers of Plasma Science at the Abdus Salam ICTP, Trieste, Italy. This work was partially supported by the Deutsche Forschungsgemeinschaft through the Sonderforschungsbereich 591 entitled 'Universal behavior of plasmas in nonequilibrium state: Heating, transport, and structure formation'.

\section{References}

[1] Yu, M. Y. and Shukla, P. K. 1978 Phys. Fluids 21, 1454. Sharma, R. P. and Shukla, P. K. 1983 Phys. Fluids 26, 87.

[2] Shukla, P. K. and Stenflo, L. 1995 Phys. Scripta T60, 32.

[3] Shukla, P. K. and Stenflo, L. 1999 Nonlinear MHD waves and Turbulence (ed. T. Passot and P. L. Sulem). Berlin: Springer, pp. 1-30.

[4] Sundqvist, D., Krasnoselskikh, V., Shukla, P. K., Vaivads, A., André, M., Buchert, S. and Rème, H. 2005 Nature 436, 825-828.

[5] Shevchenko, V. I., Galinsky, V. L., Ride, S. K. and Baine, M. 1995 Geophys. Res. Lett. 22, 2997.

Shevchenko, V., Galinsky, V., Sagdeev, R. and Winske, D. 2004 Phys. Plasmas 11, 4290.

[6] Medvedev, M. V., Diamond, P. H., Shevchenko, V. I. and Galinsky, V. L. 1997 Phys. Rev. Lett. 78, 4934.

[7] Yan, H. and Lazarian, A. 2002 Phys. Rev. Lett. 89, 281102.

Diamond, P. H. and Malkov, M. A. 2004 J. Plasma Fusion Res. Ser. 6, 28.

[8] Gekelman, W. 1999 J. Geophys. Res. 104, 14417. 
[9] Hasegawa, A. and Uberoi, C. 1982 The Alfvén Wave. Report DOE/TIC No. 11197, National Technical Information Service, US Department of Commerce, Springfield.

[10] Cramer, N. F. 2001 The Physics of Alfvén Waves. Berlin: Wiley-VCH.

[11] Shukla, P. K., Bingham, R., McKenzie, J. F. and Axford, I. 1999 Solar Phys. 186, 61. Wu, D. J. and Fang, C. 1999 Astrophys. J. 511, 958.

[12] Shukla, P. K., Bingham, R., Eliasson, B., Dieckmann, M. E. and Stenflo, L. 2006 Plasma Phys. Control. Fusion 48, B 249.

[13] Sagdeev, R. Z. and Galeev, A. A. 1969 Nonlinear Plasma Theory. New York: W. A. Benjamin.

[14] Hasegawa, A. and Chen, L. 1976 Phys. Rev. Lett. 36, 1362.

[15] Shukla, P. K. and Dawson, J. M. 1984 Astrophys. J. 276, L49.

[16] Brodin, G. and Stenflo, L. 1988 J. Plasma Phys. 39, 277; 1990 Contrib. Plasma Phys. 30, 413.

[17] Rogister, A. 1971 Phys. Fluids 14, 2733.

[18] Shukla, P. K. and Stenflo, L. 1985 Phys. Fluids 28, 1576.

Shukla, P. K., Stenflo, L., Bingham, R. and Eliasson, B. 2004 Plasma Phys. Control. Fusion 46, B349.

[19] Hollweg, J. V. 1994 J. Geophys. Res. 99, 23431.

[20] Nariyuki, Y. and Hada, T. 2006 Nonlin. Proc. Geophys. 13, 425.

[21] Nariyuki, N. and Hada, T. 2006 Preprint no:arXiv:physies/0608306 v1.

[22] Carter, T. A., Brugman, B., Pribyl, P. and Lybarger, W. 2006 Phys. Rev. Lett. 96, 155001.

[23] Kadomtsev, B. B. 1965 Plasma Turbulence. New York: Academic.

[24] Shukla, P. K., Stenflo, L. and Faria, R. T. 1998 Phys. Plasmas 5, 2846.

[25] Ichimaru, S. 1973 Basic Principles of Plasma Physics: A Statistical Approach. London: W. A. Benjamin Inc., pp. 56-58. 